\title{
The mystery of cosmic magnetogenesis
}

\author{
Christos G. Tsagas \\ Section of Astrophysics, Astronomy and Mechanics, Department of Physics \\ Aristotle University of Thessaloniki, Thessaloniki 54124, Greece \\ email: tsagas@astro.auth.gr
}

\begin{abstract}
The origin of the large-scale magnetic fields remains an open question, despite the efforts and the continual detection of magnetic fields in the universe. Primordial magnetism could answer the question of cosmic magnetogenesis, but there are still obstacles to overcome.
\end{abstract}

Keywords. magnetic fields, cosmology: theory, early universe

Magnetic fields appear to be everywhere in the universe. The Milky Way and other spiral galaxies have coherent magnetic fields of $\mu \mathrm{G}$-order magnitude, while galaxy clusters and remote protogalactic clouds also carry fields of similar strength (Han \& Wielebinski 2002, Vallée 2011). Moreover, recently, there have been independent reports of relatively strong magnetic fields (around $10^{-15} \mathrm{G}$ ) in intergalactic voids (Neronov 2010, Tavecchio et al. 2010, Ando \& Kusenko 2010). Despite this widespread presence, however, the origin of cosmic magnetism remains a mystery and an open question.

It is generally believed that the galactic fields have been amplified and sustained by some kind of dynamo action (Brandenburg \& Subramanian 2005). Dynamos, however, cannot work without an initial seed field. The latter can be the result of astrophysical processes, operating after recombination, or have primordial origin. Early-time magnetogenesis is attractive because it can in principle explain all the magnetic fields seen in the universe, especially those at high redshift and in empty intergalactic space. Nevertheless, generating primordial fields that could sustain the galactic dynamo is not a problem-free exercise (Kandus, Kunze \& Tsagas 2011). Seed fields created between the end of inflation and recombination are generally too small in size. The inverse cascade of magnetic helicity could increase the coherence length of the post-inflationary fields, but requires highly helical magnetic seeds. Inflation can solve the size problem, but magnetic fields that survived a period of standard inflation are typically too weak. Over the years there have been many attempts to solve the weakness problem of the inflationary magnetic fields. Success, however, comes at a cost. Almost all of the proposed scenarios operate outside classical electromagnetism, or introduce some other kind of new physics.

Overall, although the continual detection of magnetic fields in the universe makes the questions regarding their origin, evolution and implications increasingly pressing, the issue of cosmic magnetism remains open and the subject of ongoing debate.

\section{References}

Han, J. \& Wielebinski, R. 2002 Chin. J. Astron. Astrophys., 2, 293

Vallée, J. P. 2011 New Astron. Rev., 55, 91

Neronov, A. 2010 Science, 328, 73

Tavecchio, F., et al.2010 Mon. Not. R. Astron. Soc. 406, L70

Ando, S. \& Kusenko, A. 2010 Ap. J. Lett. 722, L39

Brandenburg, A. \& Subramanian, K. 2005 Phys. Rep. 417, 1

Kandus, A., Kunze, K. E. Tsagas, C. G. 2011 Phys. Rep., 505, 1 